\title{
Introduction to special issue: ICISA 2010
}

\author{
Alfredo Cuzzocrea • Donghwi Lee • Pascal Lorenz • \\ Yasushi Kambayashi
}

Published online: 16 November 2011

(C) Springer Science+Business Media, LLC 2011

Providing high-performance computing and security is a

performance computing systems. Second, it covers how to challenging task. High-performance computing systems and distributed environments currently suffer from security support and high-performance. This special issue addresses relationships between security and high performance systems in many aspects. First, it considers how to add security properties (authentication, authorization, accounting, confidentiality, integrity, non-repudiation, access control) to highmitigate security cost in high performance computing systems. For instance, minimizing key management and encryption/decryption cost. Third, it investigates the tradeoffs between maintaining high performance and achieving security in computing systems and solutions to balance the two objectives.

\footnotetext{
A. Cuzzocrea

ICAR-CNR and University of Calabria, Calabria, Italy

D. Lee $(\bowtie)$

University of Colorado Denver, Denver, USA

e-mail:dhclub@naver.com

P. Lorenz

Univ. de Haute Alsace, Mulhouse, France

Y. Kambayashi

Nippon Institute of Technology, Miyashiro, Japan
} 